\title{
MIOMATOSIS UTERINA E INFERTILIDAD: ¿QUÉ EVIDENCIAS TENEMOS COMO CAUSA Y COMO TRATAMIENTO?
}

\author{
Uterine fibroids and infertility: what evidence do we \\ have regarding cause and treatment? \\ Luis Ernesto Pérez, M.D.*, Ivonne Díaz, M.D.**, Luis Ospina, M.D.*** \\ Recibido: septiembre 17/07 - Aceptado: noviembre 25/08
}

\section{RESUMEN}

Objetivos: revisar las evidencias actuales que determinen el impacto de la miomatosis uterina en la infertilidad, así como la seguridad y la eficacia de las diferentes formas de tratamiento quirúrgico conservador.

Metodología: revisión sistemática cualitativa, consultando las fuentes de datos de Cochrane (Menstrual Disorders and subfertility), Medline (PubMed - MeSH), y de OVID (EBM); desde 1995 a 2008.

Resultados: muchos estudios observacionales han sugerido que los miomas causantes de infertilidad son todos los submucosos, los intramurales mayores de $5 \mathrm{~cm}$ que alteren la cavidad uterina y también los subserosos cuya cantidad y tamaño distorsionen la fisiología reproductiva. Pero no hay estudios aleatorizados controlados (RCTs) que soporten estas afirmaciones.

En la actualidad, las miomectomías constituyen la mejor forma de tratamiento para pacientes infértiles. Por histeroscopia se deben resecar miomas

* Especialista en Ginecología y Obstetricia. Profesor titular, Universidad Militar Nueva Granada, Hospital Militar Central. Bogotá, Colombia. Correo electrónico: leperez@cable.net.co

** Especialista en Ginecología y Obstetricia. Docente Unidad de Fertilidad y Endocrinología Reproductiva, Hospital Militar Central, Universidad Militar Nueva Granada y Unidad de Fertilidad Procreación Médicamente Asistida (PMA) Clínica Marly. Bogotá, Colombia.

*** Residente III Universidad del Cauca, Popayán. Con rotación por Unidad de Fertilidad y Endocrinología Reproductiva, Hospital Militar Central, Universidad Militar Nueva Granada. Bogotá, Colombia. submucosos menores de $5 \mathrm{~cm}$, tipos $0 \mathrm{y} \mathrm{I}$; por laparoscopia miomas intramurales o subserosos menores de $10 \mathrm{~cm}$ y no más de 5 miomas; finalmente, por laparotomía es posible resecar todo tipo de miomas, independientemente de su localización, tamaño y número. No hay RCTs que comparen la histeroscopia con otras técnicas de miomectomías; pero sí los hay mostrando que las indicaciones para laparotomía y laparoscopia son igualmente efectivas en resultados reproductivos.

Conclusión: hay evidencias limitadas soportando la miomatosis como causante de infertilidad, y las miomectomías por histeroscopia respecto a otros tratamientos conservadores. Sin embargo, hay RCTs que muestran iguales resultados reproductivos al realizar miomatomías intramurales o subserosas por laparoscopia y por laparotomía en pacientes seleccionadas.

Palabras clave: miomas, útero, infertilidad, miomectomías, laparotomía, histeroscopia, laparoscopia.

\section{SUMMARY}

Objectives: reviewing the evidence to determine the impact of fibroids on infertility and different types of conservative surgical treatment's efficacy and safety.

Methodology: the Cochrane Menstrual Disorders and Sub-fertility Review Group specialized register of controlled trials, MEDLINE (PUBMED-MeSH), 
and OVID (EBM) were searched from 1995 to 2008 for carrying out a qualitative systematic review.

Main results: many observational, retrospective studies have suggested that all infertility-producing myomas are submucosal, intramural myomas bigger than $5 \mathrm{~cm}$ thereby altering the endometrial cavity and subserosal myomas whose size distorts reproductive physiology; however, no randomised controlled trials (RCT) were found to support such affirmations.

Submucosal myomas must be removed by hysteroscopy (with the exception of myomas bigger than $5 \mathrm{~cm}$ or type II); however, no RCTs were found comparing hysteroscopic treatment with other types of treatment. Intramural and subserosal myomas must be removed by laparoscopy or laparotomy (depending on their localisation and size) supported by RCTs showing that there are no outcome differences in terms of reproductive results.

Conclusions: there is limited evidence for suggesting the impact of uterine myomas on infertility and the removal of submucosal myomas by hysteroscopy. Some RCTs show no differences in fertility efficacy outcome if intramural or subserosal myomas are removed via laparotomy or laparoscopy.

Key words: fibroids, infertility, myomectomy, laparotomy, hysteroscopy, laparoscopy, pregnancy rate.

\section{INTRODUCCIÓN}

La miomatosis uterina está presente entre el 20 y el $50 \%$ de las mujeres, y en algunos grupos africanos, hasta en el 70\%. Cada mioma resulta de la proliferación clonal de una célula progenitora, junto con su matriz extracelular y su tejido conectivo (fibromioma), con tres determinantes importantes: en primer lugar, existen aberraciones cromosómicas en el 40\% de las veces (brazo corto del cromosoma 6 y brazo largo del cromosoma 7, con expresión anormal del gen HMGIC); en segundo lugar, hay expresión de los factores de crecimiento TGF $\beta$, GM-CSF, IGF-I; y finalmente, el crecimiento depende del estímulo modulador de estos factores por parte de estrógenos y progesterona. ${ }^{1}$
Sin embargo, a pesar de ser tan frecuente, tan sólo del 25 al 50\% de las miomatosis ocasionan síntomas, tales como hemorragia uterina anormal, dolor pélvico, infertilidad, y los ocasionados por presión a los órganos vecinos. ${ }^{2}$ Se ha establecido que del 1 al 10\% de la infertilidad es causada por miomatosis; pero al respecto, no han sido bien entendidos el tamaño y localización de los miomas que la ocasionan, como tampoco las indicaciones de miomectomías por histeroscopia, laparoscopia o laparotomía. ${ }^{3}$

El objetivo es revisar las evidencias médicas disponibles que puedan servir de guía en infertilidad, sobre la fisiopatología de la miomatosis uterina y su mejor tratamiento.

\section{METODOLOGÍA}

Revisión sistemática cualitativa, consultando las fuentes de datos: Cochrane (Menstrual Disorders and subfertility), Medline (PubMed - MeSH), y de OVID (Evidence Based Medicine), combinado los términos: fibroids, leiomyoma, myomectomy, hysteroscopy, laparoscopy, laparotomy, surgery. La búsqueda se limitó a metaanálisis, revisiones sistemáticas y artículos de revisiones basadas en evidencias, entre 1995 y 2008. La información fue revisada, independientemente, por los dos primeros autores y al final se resumió en una tabla que muestra las jerarquías de las evidencias encontradas y sus correspondientes recomendaciones, acorde a los criterios de la Royal College of Obstetrician and Gynecologist. ${ }^{4}$

\section{MIOMATOSIS E INFERTILIDAD}

Viejos informes han asociado la miomatosis uterina con infertilidad hasta en el $27 \%$ de los casos. ${ }^{1}$ Sin embargo, varios estudios observacionales las asocian sólo en un 5 a 10\%; y más aún, después de descartar otras causas, sólo se considera la miomatosis como causa del 1 al 2,4\% de la infertilidad y del $5 \%$ del aborto recurrente. ${ }^{2,3,5}$ Los mismos estudios han determinado que los riesgos de infertilidad dependen de la localización de los miomas en el útero y de su tamaño: casi todos los submucosos 
al alterar la cavidad uterina han sido considerados como de alto riesgo; los intramurales mayores de $5 \mathrm{~cm}$ que distorsionan la cavidad uterina, como de mediano riesgo; y los subserosos con bajo riesgo, al distorsionar la anatomía y fisiología reproductiva. Los mecanismos propuestos han sido: $:^{2,3,5,6}$

1. Alteraciones del endometrio en el área de implantación del blastocisto: elongación, distorsión, alteración de la contractibilidad, inadecuada perfusión, inflamación, secreción de sustancias vasoactivas, ambiente androgénico y alteraciones del metabolismo del calcio.

2. Interferencia con el transporte de gametos: distorsión anatómica o funcional de cavidad uterina y trompas.

3. Impedimento anatómico y de perfusión sanguínea para la progresión del embarazo.

Pritts, en una revisión sistemática de 11 estudios de cohortes retrospectivos, ${ }^{7}$ mostró que la miomatosis submucosa conlleva a menores tasas de embarazo, al compararla con otras causas de infertilidad ( $\mathrm{RR} 0,3$; Cl 0,13 - 0,70); las miomectomías no incrementaron las tasas de recién nacidos vivos (RR 0,98; 95\% Cl 0,45 - 2,91), pero sí las tasas de embarazos (RR 1,72; 95\% Cl 1,13-2,58); además, los miomas intramurales, que alteraron la cavidad uterina, redujeron también las probabilidades de embarazo, al compararlos con mujeres sin miomas llevadas a tratamientos de reproducción asistida (RR 0,46; 95\% Cl 0,24-0,88) [evidencia II-b].

Hasta 1999, cinco estudios de cohorte retrospectivos $^{8-12}$ habían mostrando, en programas de fertilización in vitro (FIV), el impacto de la miomatosis submucosa e intramural, con tasas de embarazos por transferencia de 9 a 16\%, y 37\% para miomas submucosos, intramurales y subserosos respectivamente; comparados con tasas de embarazo de 30\% en los grupos de control sin miomas, [evidencias II-b]. En el 2001, un estudio de cohortes prospectivo, por Hart R y col. ${ }^{13}$ mostró que pacientes con miomas intramurales llevadas a FIV reducen sus tasas de embarazos al compararlas con pacientes sin miomas (OR 0,46; 95\% CI 0,24 a 0,88), una vez rea- lizados ajustes a número de embriones transferidos y edad de las pacientes [evidencia II-a]. En el 2002, otro estudio de cohorte prospectivo ${ }^{14}$ determinó, en pacientes por FIV, que los miomas intramurales pequeños, menores de $5 \mathrm{~cm}$ y que no deforman la cavidad uterina, no alteran significativamente las tasas de embarazos (34,4\% vs. $47,5 \%)$, ni las tasas de partos (22,9\% vs. 37,7\%) [evidencia II-a].

En el 2004, en una serie de casos de FIV, ${ }^{15}$ Wallach y col. relacionaron los miomas que deforman la cavidad uterina con bajas tasas de embarazos y altas de abortos (hasta del 50\%), e informaron mejoría de estas tasas al practicar miomectomías [evidencia III]. Por otra parte, Surrey y col. en el 2005, en una serie de casos y controles ${ }^{16}$ en programas de FIV, con y sin donación de oocitos, compararon tasas de embarazo en tres grupos: receptoras post-resección de miomas submucosos por histeroscopia, receptoras post-resección de miomas intramurales por laparotomía, y receptoras sin miomas como controles; y mostraron tasas de embarazo similares en los tres grupos, tanto de donación de oocitos (86,7\% - 84,6\% - 77\%) como de FIV (61\% - 52\% - 53\%); concluyendo que, en pacientes seleccionadas, las resecciones de los miomas producen similares tasas de embarazo que en los controles sin miomas ${ }^{15}$ [evidencia II-b].

En el 2006, Ballesteros y col. ${ }^{17}$ también en casos y controles de 65 ciclos de FIV con miomas, versus 366 de FIV sin miomas, reafirmaron que los miomas intramurales menores de $5 \mathrm{~cm}$, que no distorsionan la cavidad uterina, no alteran las tasas de embarazos (20\% vs. 23\%), abortos (46,1\% vs. $26,4 \%$ ), ni de recién nacidos ( $46,1 \%$ vs. $56,8 \%$ ), por lo que se cuestionaron las miomectomías en estas circunstancias [evidencia II-b].

Las miomectomías por histeroscopia, laparoscopia o laparotomía, son hasta ahora la mejor alternativa para el manejo de la miomatosis en pacientes infértiles. ${ }^{18-20}$ La embolización de arterias uterinas no es una alternativa en infertilidad, debido a que estudios observacionales y RCTs, muestran entre sus complicaciones falla ovárica hasta en el 14\%. ${ }^{21,22}$ Ver resumen de la evidencia en la tabla 1. 
Tabla 1. Mejores evidencias en miomatosis uterina e infertilidad.

\begin{tabular}{|c|c|c|}
\hline & Jerarquía & Recomendación \\
\hline Infertilidad por miomatosis uterina: $1 \%-2,4 \%$ & II-b y III & \\
\hline \multicolumn{3}{|l|}{ Miomas submucosos } \\
\hline Causan infertilidad al alterar la cavidad uterina & II-a & \\
\hline $\begin{array}{l}\text { Resección por histeroscopia si son menores de } 4-5 \mathrm{~cm} \text {, tipos } 0 \text { y } \\
\text { I, y tipo II si hay habilidad quirúrgica }\end{array}$ & II-b & $\mathrm{B}$ \\
\hline $\begin{array}{l}\text { Resección por laparotomía los tipo II y los mayores de } 5 \text { cm de } \\
\text { todos los tipos }\end{array}$ & III & $\mathrm{C}$ \\
\hline
\end{tabular}

\section{Miomas intramurales}

Sólo causan infertilidad si son mayores de $5 \mathrm{~cm}$, y si afectan la cavidad uterina

II-a

Igual resección por laparoscopia o por laparotomía, si son menores de $10 \mathrm{~cm}$ y menos de 5 miomas

\section{Miomas subserosos}

Causan infertilidad si distorsionan la fisiología reproductiva

Resección por laparoscopia o por laparotomía, igual que intramurales

\section{Coadyuvantes quirúrgicos útiles}

GnRHa previos

Vasopresina o misoprostol, intraoperatorios

Gel de ácido hialurónico para prevenir adherencias

\section{MIOMECTOMÍAS POR HISTEROSCOPIA}

Varios estudios de series de casos retrospectivos y de presentación de $\operatorname{casos}^{23-28}$ [evidencia III] muestran en más de 200 pacientes infértiles, a quienes se les resecaron miomas submucosos con tamaños entre 1 y $6 \mathrm{~cm}$, tasas de embarazo de 55\%, de los cuales el $80 \%$ nacieron vivos a término.

Los miomas submucosos son de tres tipos: 0, intracavitarios; I, más del 50\% del mioma intracavitario; y II, más del 50\% del mioma intramural. ${ }^{26}$ Los mejores resultados se notaron post-resección de miomas tipo 0; pero similares en tasas de embarazos, al resecarlos por laparotomía. Sus posibles ventajas son: técnica menos invasiva, bajo riesgo de ruptura de la cicatriz uterina durante el embarazo, y menor formación de adherencias intracavitarias que con laparotomía. También advierten que la resección de miomas tipo II, requiere mayor habilidad quirúrgica, y los grandes, varias sesiones. Sugieren que todo mioma submucoso mayor de 4 - $5 \mathrm{~cm}$, de cualquier tipo, debe ser resecado por laparotomía; por histeroscopia los menores de $5 \mathrm{~cm}$, tipos 0 y I; y los tipo II sólo en manos de un cirujano muy experto, que evite la perforación uterina. ${ }^{26}$

En el 2005, Shokeir ${ }^{29}$ publicó un estudio prospectivo de una serie de casos de 29 pacientes infértiles, por causa de miomatosis. Resecó miomas submucosos tipo I y II, menores de $5 \mathrm{~cm}$, y mostró cómo las tasas de parto pasaron de 3,8\% a 63,2\%; y las de aborto de $61,6 \%$ a $26,3 \%$ [evidencia III].

Finalmente en el 2007, Makris y col. ${ }^{30}$ utilizando el resectoscopio bipolar, realizaron 15 miomectomías tipo 0, 34 tipo I y 10 tipo II, en un grupo de subfértiles e infértiles por miomatosis. Los tamaños de los miomas fueron 15 t/- $10 \mathrm{~mm}$. Con el seguimiento mostraron los beneficios de los 
procedimientos, al obtener $42,4 \%$ de embarazos en todas, y 54,16\% en quienes tenía la miomatosis como única causa de infertilidad con miomas de $25 \mathrm{~mm}$. No se encontraron RCTs que comparen el manejo histeroscópico frente a otros tipos de manejo de los miomas. ${ }^{31}$

\section{MIOMECTOMÍAS POR LAPAROSCOPIA}

La resección de miomas por laparoscopia en pacientes infértiles, amerita mejor selección de las pacientes y mayor experticia del cirujano; más aún cuando se afirma: "por laparoscopia, generalmente, se resecan miomas que no afectan para nada la fertilidad, pero sí la comprometen con síndrome adherencial futuro". Además, existe el riesgo de ruptura de cicatriz uterina durante el embarazo y el trabajo de parto; ${ }^{32}$ así como la formación de protrusiones en el sitio de la laparoscopia y formación de fístulas. ${ }^{33}$ Afortunadamente, han ido apareciendo mejores equipos de laparoscopia, mejor instrumental (morceladores electro-mecánicos, bisturí armónico, grapadores lineales, etc.), se ha ido mejorando la técnica y la experiencia quirúrgica del cirujano, pero también la mejor selección de las pacientes.

En el 2003, Malzony y col..$^{34}$ publicaron una serie de 144 casos [evidencia III] con miomas intramurales y subserosos entre 5 y $18 \mathrm{~cm}$, en pacientes con infertilidad (70,8\%), hemorragia uterina anormal y dolor pélvico. Mostraron post-remoción laparoscópica, tasas de embarazos de 55,26\% en 12 meses de seguimiento, franca disminución de la hemorragia, y sustancial alivio del dolor, respectivamente, en cada grupo estudiado. Complicaciones 2,08\%: dos casos fueron convertidas a laparotomía, una ameritó transfusión sanguínea y no hubo rupturas uterinas durante el embarazo. Promedio de hospitalización 2,6 días.

También en el 2003, Campo y col. en un estudio de cohortes prospectivo ${ }^{35}$ [evidencia II-a], compararon la resección de miomas intramurales y subserosos en pacientes infértiles, 9 por laparotomía y 22 por laparoscopia; mostrando tasas de embarazo de $60,9 \%$ y de abortos $13,8 \%$; similares en ambas técnicas.

Otro estudio multicéntrico retrospectivo, ${ }^{36}$ comparó 126 miomectomías por laparoscopia, contra 176 por laparotomía. Mostró a favor de la laparoscopia menor tiempo de recuperación; y en contra, dificultad para resecar miomas mayores de $10 \mathrm{~cm}$ y más de 5 miomas, localizados en la pared uterina anterior o en la región intersticial; hubo conversión a laparotomía en el 29\% de los casos. La conclusión fue que la ecografía preoperatoria es fundamental para determinar número, localización y tamaño de los miomas, a fin de ofrecer la mejor alternativa quirúrgica.

En el año 2000, el RCT de Seracchiolli y col. ${ }^{37}$ [evidencia I-b] muestra que, tanto las miomectomías por laparotomía como por laparoscopia son sensiblemente iguales, con tasas de embarazos de 55,9 y 53,6\%; y tasas de abortos de 12 y $20 \%$ respectivamente. Se diferenciaron solamente en menor sangrado por laparoscopia y mayor morbilidad febril y hemorrágica por laparotomía.

Las recurrencias de miomatosis también son similares, tanto para laparoscopia como para laparotomía; ${ }^{38}$ obviamente no dependen de la técnica quirúrgica, excepto que se dejen de resecar los pequeños por dificultad en palparlos o encontrarlos, porque las recidivas dependen de cada paciente con su genética, factores de crecimiento y esteroides gonadales.

En el 2006, el RCT de Alessandri y col. ${ }^{39}$ [evidencia Ib-A] compara la laparoscopia frente a la minilaparotomía, en 149 pacientes. Encontró a favor de la minilaparotomía, menor tiempo quirúrgico $(\mathrm{p}<0,001)$ y menos costos $(1975$ euros vs. 2250 euros); y a favor de la laparoscopia, menos sangrado y menor tiempo de recuperación, pero en dos hubo perforación y peritonitis. Además, en el 2007 Palomba y col. en un RCT multicéntrico ${ }^{40}$ [evidencia Ib-A] con 136 pacientes, concluyeron similares resultados con las dos técnicas, pero enfatizaron en la necesidad de seleccionar las pacientes aptas para cada una de ellas. 


\section{MIOMECTOMÍAS POR LAPAROTOMÍA}

En 1998, Versellini y col. hacen una revisión de las miomectomías por laparotomía, en 27 artículos de series de casos (sólo 9 prospectivos), que suman un total de 138 infértiles por miomatosis ${ }^{41}$ [evidencia III- C]. El seguimiento por más de 12 meses demostró tasas de embarazos de $57 \%$ en el promedio de todos, de $61 \%$ en los estudios prospectivos, de 58 a $65 \%$ si la miomatosis era intramural o subserosa, y de 53 a 70\% si era intramural. Concluyó que 2/3 de las pacientes operadas por laparotomía lograban embarazarse. Hasta hoy en día, se han publicado varias series de casos referentes a la sola laparotomía ${ }^{2}$ y los RCTs antes mencionados, comparándola con la laparoscopia. ${ }^{36-40}$

Los estudios sugieren que por laparotomía se pueden realizar todo tipo de miomectomías, independientemente de su localización, tamaño y número. Pero cuando se tiene el recurso de histeroscopia y laparoscopia, y sus ventajas aparte de los resultados reproductivos, se deben seleccionar para miomectomías por laparotomía, pacientes con miomas submucosos con tamaños mayores de 4-5 cm, con más de 5 miomas intramurales, y con miomas intramurales y subserosos mayores de 10 $\mathrm{cm}$; así como los localizados en el istmo anterior y las regiones intersticiales, dada la dificultad y el riesgo de sangrado por laparoscopia. La complicación futura más relacionada ha sido la formación de adherencias pélvicas, proporcional al traumatismo de la técnica quirúrgica.

\section{COADYUVANTES DE LAS MIOMECTOMÍAS}

En la actualidad, para las miomectomías por laparoscopia o laparotomía, hay tres coadyuvantes a tener en cuenta:

Agonistas de la hormona liberadora de gonadotrofinas (GnRHa): la mejor evidencia de su utilidad se deriva de la revisión sistemática del grupo Cochrane ${ }^{42}$ en 20 RCTs, que comparó los GnRHa con controles o placebo. Se encontraron beneficios significativos cuando se utilizan por 3 ó 4 meses en el preoperatorio: reducción del tamaño de los miomas y del útero, reducción de la anemia ferropénica preoperatoria, así como reducción de las pérdidas de sangre intraoperatoria.

\section{Reductores de hemorragia intraoperatoria:} las revisiones sistemáticas del grupo Cochrane en el $2003^{43}$ y el $2007^{44}$ en 8 RCTs, muestran reducción significativa de la hemorragia intraoperatoria al utilizar: a) misoprostol (diferencias ponderadas de la media (DPM) -149,00 ml; 95\% CI -229,24 a $68,76)$, b) vasopresina y análogos (DPM -298,72 ml; 95\% CI -593,10 a -4,34), c) torniquete pericervical (DPM -1870,00 ml; 95\% CI -2547,16 a -1192,84), y d) disección con mesna (Mesnex®) $(\mathrm{P}<0,05)$. Pero sin beneficios bupivacaina más epinefrina (DPM -68,60 ml; 95\% CI -93,69 a - 43,51), aplicación de oxitocina, ni morcelación in situ.

Reductores del riesgo de adherencias: todas las barreras antiadherenciales han sido consideradas inefectivas para este propósito, excepto la buena técnica quirúrgica con manejo adecuado de la hemostasia y la aplicación de gel de ácido hialurónico sobre áreas cruentas, a la que un $\mathrm{RCT}^{45}$ le encontró beneficios significativos.

\section{CONCLUSIÓN}

La evidencia que soporta las implicaciones de la miomatosis uterina en infertilidad, así como la eficacia de la histeroscopia, es débil por falta de estudios experimentales (RCTs) bien elaborados, que reduzcan el riesgo de sesgos, especialmente comparando localizaciones y tamaños de miomas en pacientes infértiles, con pacientes fértiles ${ }^{44,45} \mathrm{en}$ el primer caso, y que comparen la histeroscopia con otras formas de manejo, incluyendo el expectante en pacientes con miomas submucosos menores de 2 $\mathrm{cm} .{ }^{30}$ Sólo hay recomendaciones B para resecar por este medio los miomas, 0 y I, menores de $5 \mathrm{~cm}$; y recomendación C para los tipo II, dependiendo de la habilidad del cirujano. Según lo anterior, los miomas submucosos mayores de $5 \mathrm{~cm}$ siempre deberían ser resecados por laparotomía. 
La evidencia soporta el hecho, según el cual la miomectomía intramural y subserosa por vía laparoscópica o por laparotomía, ofrece iguales resultados reproductivos en manos expertas, que seleccionen bien a las pacientes infértiles para cada una de estas dos técnicas. ${ }^{31}$ La decisión de cuál de las dos técnicas se debe tomar, para cada paciente en particular, dependerá de la experiencia del cirujano y de la disponibilidad de los equipos apropiados.

Por otra parte, y para finalizar; con recomendación A se justifica el empleo preoperatorio de análogos de la GnRH, para reducir tamaño y sangrado peoperatorio; la vasopresina o el misoprostol, para reducir la hemorragia intraoperatoria; y el uso de gel de ácido hialurónico, para reducir el riesgo de adherencias.

\section{REFERENCIAS}

1. Bukulmez O, Doody KJ. Clinical features of myomas. Obstet Gynecol Clin North Am 2006;33:69-84.

2. Bajekal N, Li TC. Fibroids, infertility and pregnancy wastage. Hum Reprod Update 2000;6:614-20.

3. Practice Committee of the American Society for Reproductive Medicine. Myomas and reproductive function. Fertil Steril 2006;86:S194-9.

4. Royal College of Obstetrician and Gynecologist. Fertility: assessment and treatment for people with fertility problems. Clinical guideline. London: RCOG press; feb 2004.

5. Vercellini P, Maddalena S, De Giorgi O, Pesole A, Ferrari L, Crosignani PG. Determinants of reproductive outcome after abdominal myomectomy for infertility. Fertil Steril 1999;72:109-14.

6. Sozen I, Arici A. Cellular biology of myomas: interaction of sex steroids with cytokines and growth factors. Obstet Gynecol Clin North Am 2006;33:41-58.

7. Pritts EA. Fibroids and infertility: a systematic review of the evidence. Obstet Gynecol Surv 2001;56:483-91.

8. Seoud MA, Patterson R, Mausher SJ, Coddington CC 3rd. Effects of myomas or prior myomectomy on in vitro fertilization performance. J Assist Reprod Genet 1992;9:217-21.

9. Farhi J, Ashkenazi J, Feldberg D, Dicker D, Orvieto R, Ben Rafael Z. Effect of uterine leiomyomata on the results of in vitro fertilization treatment. Hum Reprod 1995;10:2576-8.
10. Eldar-Geva T, Meagher S, Healy DL, MacLachlan V, Breheny S, Wood C. Effect of intramural, subserosal, and submucosal uterine fibroids on the outcome of assisted reproductive technology treatment. Fertil Steril 1998;70:687-91.

11. Stovall DW, Parrish SB, Van Voorhis BJ, Hahn SJ, Sparks AE, Syrop CH. Uterine leiomyomas reduce the efficacy of assisted reproduction cycles: results of a matched follow-up study. Hum Reprod 1998;13:192-7.

12. Ramzy AM, Sattar M, Amin Y, Mansour RT, Serour GI, Aboulghar MA. Uterine myomata and outcome of assisted reproduction. Hum Reprod 1998;13:198-202.

13. Hart R, Khalaf Y, Yeong CT, Seed P, Taylor A, Braude P. A prospective controlled study of the effect of intramural uterine fibroids on the outcome of assisted conception. Hum Reprod 2001;16:2411-7.

14. Check JH, Choe JK, Lee G, Dietterich C. the effect on IVF outcomes of small intramural fibroids not compressing the uterine cavity as determined by a prospective matched control study. Hum Reprod 2002; 17:1244-8.

15. Wallach E, Vlahos N F. Uterine myomas: an overview of development, clinical features, and management. Obstet Gynecol 2004;104:393-406.

16. Surrey ES, Minjarez DA, Stevens JM, Schoolcraft WB. Effect of myomectomy on the outcome of assisted reproductive technologies. Fertil Steril 2005;83:1473-9.

17. Ballesteros Manzo A. Barros Delgadillo JC. Ochoa Rueda S. Villalobos Acosta S. Barroso Villa G. Sánchez Solis V. Gavino Gavino F. Efecto de los miomas intramurales y subserosos en los ciclos de fertilizacion in vitro y sus resultados perinatales. Ginecología y Obstetricia de México 2006;74:55-65.

18. Olive DL, Lindheim SR, Pritts EA. Non-surgical management of leiomyoma: impact on fertility. Curr Opin Obstet Gynecol 2004;16:239-43.

19. Olive DL, Lindheim SR, Pritts EA. Conservative surgical management of uterine myomas. Obstet Gynecol Clin North Am 2006;33:115-24.

20. Pérez LE. Miomectomías: indicaciones y técnicas quirúrgicas, una visión actual. Controv en Ginecol y Obstet. 2008;19:3676-85.

21. Gupta JK, Sinha AS, Lumsden MA, Hickey M. Uterine artery embolization for symptomatic uterine fibroids that cause symptoms such as heavy bleeding, pain, pressure symptoms and subfertility. Cochrane Database of Systematic Reviews 2006, Issue 1. Art. No.: CD005073. 
22. Goodwin SC, Bradley LD, Lipman JC, Stewart EA, Nosher JL, Sterling KM, et al. Uterine artery embolization versus myomectomy: a multicenter comparative study. Fertil Steril 2006;85:14-21.

23. Hallez JP. Single-stage total hysteroscopic myiomectomies; indications, thecniques and results. Fertil Steril 1995;63:703-8.

24. Giatras K, Berkeley AS, Noyes N, Licciardi F, Lolis D, Grifo JA, et al. Fertility alter hysteroscopic resection of submucos myomas. J Am Assoc Gynecol Laparosc 1999;6:155-8

25. Vercellini P, Zaina B, Yaylayan L, Pisacreta A, De Giorgi O, Crosignani PG. Hysteroscopic myomectomy: long term effects of menstrual pattern and fertility. Obstet Gynecol 1999;94:341-7.

26. Hucke J, De Bruyne F, Balan P. Hysteroscopy in infertility: diagnosis and treatment including falloposcopy. Review. Contrib Gynecol Obstet 2000;20:13-20.

27. Fernández H, Kadoch O, Capella-Allouc S, Gervaise A, Taylor S, Frydman R. Hysteroscopic resection of submucous myomas: long term results. Ann Chir 2001;126:58-64.

28. Pérez LE, Arboleda W. Complicaciones en histeroscopia, a propósito de un caso. Controv en Ginecol y Obstet. 2005;14:2062-8.

29. Shokeir TA. Hysteroscopic management in submucous fibroids to improve fertility. Arch Gynecol Obstet 2005;273:50-4.

30. Makris N, Vomvolaki E, Mantzaris G, Kalmantis K, Hatzipappas J, Antsaklis A. Role of a bipolar resectoscope in subfertile women with submucous myomas and menstrual disorders. J Obstet Gynaecol Res 2007;33:849-54.

31. Griffiths A, D'Angelo A, Amso N. Surgical treatment of fibroids for subfertility. Cochrane Database Systematic Review 2006, Issue 3. Art. No.: CD003857.

32. Dubuisson JB, Chavet X, Chapron C, Gregorakis SS, Morice P. Uterine rupture during the pregnancy after laparoscopic myomectomy. Hum Reprod 1995;10:1475-7.

33. Nezhat C. Laparoscopic myomectomy complication. Int J Fertil 1992;37:64.

34. Malzoni M, Rotond M, Perone C, Labriola D, Ammaturo F, Izzo A, et al. Fertility after laparoscopic myomectomy of large uterine myomas: operative technique and preliminary results. Eur J Gynaecol Oncol 2003;24:79-82.

35. Campo S, Campo V, Gambadauro P. Reproductive outcome before and after laparoscopic or abdominal myomectomy for subserous or intramural myomas. Eur J Obstet Gynecol Reprod Biol 2003;110:215-9.

36. Marret H, Chevillot M, Giraudeau B; Study Group of the French Society of Gynaecology and Obstetrics (Ouest Division). A retrospective multicentre study comparing myomectomy by laparoscopy and laparotomy in current surgical practice. What are the best patient selection criteria? Eur J Obstet Gynecol Reprod Biol 2004;117:82-6

37. Seracchioli R, Rossi S, Govoni F, Rossi E, Venturoli S, Bulletti C, et al. Fertility and obstetric outcome after laparoscopic myomectomy of large myomata: a randomized comparison with abdominal myomectomy. Hum Reprod 2000;15:2663-8.

38. Rossetti A, Sizzi O, Soranna L, Cucinelli F, Mancuso $\mathrm{S}$, Lanzone A. Long-term results of laparoscopic myomectomy: recurrence rate in comparison with abdominal myomectomy. Hum Reprod 2001;16:770-4.

39. Alessandri F, Lijoi D, Mistrangelo E, Ferrero S, Ragni N. Randomized study of laparoscopic versus minilaparotomic myomectomy for uterine myomas. J Minim Invasive Gynecol 2006;13:92-7.

40. Palomba S, Zupi E, Russo T, Falbo A, Marconi D, Tolino A, et al. A multicenter randomized, controlled study comparing laparoscopic versus minilaparotomic myomectomy: short-term outcomes. Fertil Steril 2007;88:942-51.

41. Vercellini P, Maddalena S, De Giorgi O, Aimi G, Crosignani PG. Abdominal myomectomy for infertility: a comprehensive review. Hum Reprod 1998;13:873-9.

42. Lethaby A, Vollenhoven B, Sowter M. Pre-operative GnRH analogue therapy before hysterectomy or myomectomy for uterine fibroids. Cochrane Database of Systematic Reviews 2000, Issue 1. Art. No.: CD000547.

43. Pellicano M, Bramante S, Cirillo D, Palomba S, Bifulco G, Zullo F, et al. Effectiveness of autocrosslinked hyaluronic acid gel after laparoscopic myomectomy in infertile patients: a prospective, randomized, controlled study. Fertil Steril 2003;80:441-4.

44. Kongnyuy EJ, Wiysonge CS. Interventions to reduce haemorrhage during myomectomy for fibroids. Cochrane Database of Systematic Reviews 2007, Issue 1. Art. No.: CD005355.

45. Diamond MP. Reduction of adhesions after uterine myomectomy by Seprafilm membrane (HAL-F): a blinded, prospective, randomized, multicenter clinical study. Seprafilm Adhesion Study Group. Fertil Steril 1996;66:904. 